\title{
Neural correlates of a single-session massage treatment
}

\author{
D. Sliz • A. Smith $\cdot$ C. Wiebking $\cdot$ G. Northoff $\cdot$ S. Hayley
}

Published online: 20 January 2012

(C) The Author(s) 2012. This article is published with open access at Springerlink.com

\begin{abstract}
The current study investigated the immediate neurophysiological effects of different types of massage in healthy adults using functional magnetic resonance imaging (fMRI). Much attention has been given to the default mode network, a set of brain regions showing greater activity in the resting state. These regions (i.e. insula, posterior and anterior cingulate, inferior parietal and medial prefrontal cortices) have been postulated to be involved in the neural correlates of consciousness, specifically in arousal and awareness. We posit that massage would modulate these same regions given the benefits and pleasant affective properties of touch. To this end, healthy participants were randomly assigned to one of four conditions: 1. Swedish massage, 2. reflexology, 3. massage with an object or 4. a resting control condition. The right foot was massaged while each participant performed a cognitive
\end{abstract}

Electronic supplementary material The online version of this article (doi:10.1007/s11682-011-9146-z) contains supplementary material, which is available to authorized users.

D. Sliz $\cdot$ S. Hayley $(\bowtie)$

Department of Neuroscience, Carleton University,

1125 Colonel By Drive,

Ottawa, ON, Canada K1S 5B6

e-mail: shayley@ccs.carleton.ca

A. Smith

Department of Psychology, University of Ottawa,

Ottawa, ON, Canada

C. Wiebking $\cdot$ G. Northoff

Mind, Brain Imaging and Neuroethics,

Institute of Mental Health Research, University of Ottawa,

Ottawa, ON, Canada

\section{Wiebking}

Department of Psychiatry, Otto-von-Guericke,

University of Magdeburg,

Magdeburg, Germany association task in the scanner. We found that the Swedish massage treatment activated the subgenual anterior and retrosplenial/posterior cingulate cortices. This increased blood oxygen level dependent (BOLD) signal was maintained only in the former brain region during performance of the cognitive task. Interestingly, the reflexology massage condition selectively affected the retrosplenial/posterior cingulate in the resting state, whereas massage with the object augmented the BOLD response in this region during the cognitive task performance. These findings should have implications for better understanding how alternative treatments might affect resting state neural activity and could ultimately be important for devising new targets in the management of mood disorders.

Keywords Touch $\cdot$ Tactile stimulation $\cdot \mathrm{fMRI} \cdot$ Default mode network $\cdot$ Interoception $\cdot$ Anterior cingulate $\cdot$ Posterior cingulate $\cdot$ Resting state

\section{Introduction}

Massage therapy is a billion dollar industry in North America with approximately $8 \%$ (over four million) of North Americans using this complementary therapy on a yearly basis (Barnes et al. 2008; Park 2005). In 2007, it was estimated that out of pocket expenses for CAM therapies cost $\$ 11.9$ billion dollars with over $\$ 4$ million being spent on massage therapy (Nahin et al. 2009). Its use has steadily risen over the last decade, and is often used as an adjunct treatment for a number of conditions, including those involving chronic pain and distress (Sherman et al. 2009; Field 2002), as well as enhancing growth and cognitive functioning in preterm infants (Field et al. 2010; Field et al. 2008; Procianoy et al. 2010) and preschoolers (Hart et al. 1998). Emerging evidence has also indicated that 
massage might have beneficial effects in cancer patients by modulating immunity, and lessening pain and sleep disturbances (Russell et al. 2008; Hughes et al. 2008; Calenda 2006). In fact, 45 min of Swedish massage was reported to increase the number of circulating $\mathrm{T}$ lymphocytes, while reducing mitogen induced inflammatory cytokine responses and limiting hormonal variations (Rapaport et al. 2010). Yet, such findings have not been uniformly accepted, as others have failed to find such effects. For instance, a recent report revealed that 5 weeks of 30 min weekly massage did not affect peripheral cytokine or $\mathrm{T}$ lymphocyte responses in a group of female breast cancer patients (Krohn et al. 2011). Of particular interest to the present investigation, a recent meta-analysis (17 different studies) demonstrated that massage therapy alleviated depressive symptoms (Hou et al. 2010). Similarly, others have found that massage reduced physiological and psychological aspects of anxiety in several different populations (Field et al. 1996; Beider et al. 2007; Moyer et al. 2004).

Despite the increasing research in complementary treatments such as massage, few studies have examined the neurobiological correlates of massage therapy. Of these, one study involving depressed adolescents who received a 15-min chair massage resulted in lessened right frontal EEG activation, which is known to be involved in negative affect and withdrawal tendencies (Jones and Field 1999). A recent PET study involving healthy participants, revealed that 4 min of a back massage increased cerebral blood flow (rCBF) in the left precuneus and pons and a subsequent increase was evident in bilateral precuneus and left fusiform gyrus after a further $20 \mathrm{~min}$ of the massage (Ouchi et al. 2006). Remarkably however, there are presently no experimental fMRI data regarding the pattern of brain region activation in response to massage therapy. To this end, the present investigation sought to evaluate the neural correlates of an acute massage session. In particular, we focus upon two brain regions that are known to be important for emotional processing and comprise critical nodes in the default mode network, the subgenual anterior cingulate cortex (sACC) and retrosplenial/posterior cingulate cortex (RSC/PCC).

Over the last decade, the resting state of the brain, which has been referred to as the default mode network (DMN; Raichle et al. 2001), has received much attention for its importance in the generation of consciousness (Greicius et al. 2008; Vanhaudenhuyse et al. 2010). The DMN encompasses a set of neural networks which show positive blood oxygen level dependant (BOLD) responses when the brain is at rest (i.e. when one is sitting quietly with eyes closed and not engaged in any task) and has been postulated to play a role in self-relatedness (Schneider et al. 2008) and subjective awareness (Khalsa et al. 2009). Among the regions of the DMN, the anterior and posterior cingulate cortices (ACC and $\mathrm{PCC}$, respectively) show a predominantly negative
BOLD response during stimulus-induced activity and have been closely associated with self-relatedness, which refers to internal and external states that a person identifies with (i.e. one's heart rate, self-consciousness) (Northoff et al. 2010; Vanhaudenhuyse et al. 2011). Importantly, the ACC and $\mathrm{PCC}$, in conjunction with the insula, are also thought to mediate changes of the peripheral autonomic nervous system and our sensation and perception of these physiological changes, which is known as interoception (Berlucchi and Aglioti 2010; Craig 2002; Wiens 2005). These changes are likely important nodes in the neural circuitry responsible for processing emotionally relevant information (Taylor et al. 2009). In fact, the closely related subgenual cingulate brain region is the site of deep brain stimulation used clinically for treatment resistant depression (Johansen-Berg et al. 2008).

Since massage involves the application of an external, primarily non-self-related stimulus, it raises the question of how it might impact the PCC and ACC and default mode processing in general. The following study will elucidate how massage can differentially regulate these two brain regions at rest and during the performance of a cognitive task (i.e. Go/No Go). In effect, we aim to test whether massage might modulate the activity of neural circuits important for maintaining resting state and general arousal.

\section{Methods}

\section{Participants}

Healthy participants were recruited within the greater Ottawa region of Ontario, Canada $(N=42)$. Informed consent was obtained as approved by the Ottawa Hospital Research Ethics Internal Review Board and the Carleton University Ethics Committee for Psychological Research. Participants were screened to ensure that they satisfied MRI safety requirements. Exclusion criteria included scores $\geq 17$ on the 21 -item Beck Depression Inventory (BDI; Beck et al. 1961). The BDI was administered to ensure that our sample did not express any depressive tendencies. The BDI is a 21 -item self-report inventory designed to measure the severity of depressive symptomatology. According to Beck et al. (1988), the cut-off points for the BDI were classified as follows: minimal or none (0-9), mild (10-16), moderate (17-29), and severe (30-63). The BDI score correlates significantly with diagnoses of clinical depression and it has well-established psychometric properties in both psychiatric and non-psychiatric samples (Beck et al. 1988).

We used a structured interview to exclude participants with major DSM-IV-TR Axis I disorders (current bipolar disorder, panic disorder, borderline personality disorder, obsessive-compulsive disorder, phobia, schizophrenia, eating disorder, post-traumatic stress disorder, suicidality beyond simple ideation or had made previous attempts, 
alcohol/substance abuse within the last 3 months, current or past psychosis, manic/hypomanic episodes, serious medical illness). Since this interview amounted to asking the subject whether he/she had any of these conditions, we cannot claim to have clinically assessed any of the subjects. Thus, we acknowledge the caveat that some subjects may actually be unaware of having a disorder or alternatively, might be lying to the experimenter.

Contraindications to massage therapy were screened, once again, via a structured interview (i.e. recent medical surgeries in the last 3 months, transplant surgery, electronic implants, heart condition, epilepsy, diabetes [in hypoglycemic state], deep vein thrombosis, high or low blood pressure, cancer, verrucae, Athlete's foot, nail fungus on feet, renal failure, bone involvement and resulting complications on affected joints; arthritis/arthrosis; angina pectoris; myocardial infarction; phlebitis; hemophilia; cerebrovascular accident). Participants needed to have had previous experience with massage therapy in order to be familiar and comfortable with the foot treatments they would be receiving in the fMRI scanner. They needed to have had received a minimum of three massage therapy treatments in their lifetime which needed to be professionally administered by a registered massage therapist. One participant was excluded from analyses due to a high score (over 17) on the BDI, indicating depressive tendencies and another participant's brain scans were excluded due to motion artifact. In total, fMRI data from 40 healthy individuals were used for this study. The participants were right-handed (12 male, 28 female) ranging in age from 20 to 62 years (mean $(M)=32.33$, standard deviation $(\mathrm{SD})=9.78)$. Each participant, on the day of testing, was randomly assigned to a tactile condition (massage with a wooden object ( 7 females and 2 males; mean age: 37.6, $\mathrm{SD}=14.0$ ), Swedish foot massage ( 6 females and 4 males; mean age: $32.1, \mathrm{SD}=9.31$ ), reflexology (7 females and 4 males; mean age: $30.3, \mathrm{SD}=6.65$ ) or the control condition (which was a non-tactile manipulation, i.e. participants lay on the scanner bed undisturbed and performed the cognitive task, 8 females and 2 males; mean age: $30.1, \mathrm{SD}=8.13$ ). Participants were not aware of which group they would be assigned to prior to being scanned. The groups were not significantly different across gender distribution or age.

\section{Behavioral measures}

Prior to the scanning session, each participant completed questionnaires assessing overall mood state and depressive tendency. The Positive Affect Negative Affect Scale (PANAS; Watson et al. 1988) is a 41-item scale made up of several emotion-based constructs. The PANAS measure has positive and negative affect sub-scales (10 items each) which evaluate one's subjective degree of unpleasurable (i.e. upset, scared, irritable) and pleasurable (i.e. enthusiastic, alert, attentive) feelings, respectively. Participants are asked to rate a series of single-word adjectives on a 7-point scale $(0=$ not at all, $6=$ extremely) indicating the extent to which that emotion is present during their daily life. In order to assess each participant's subjective experience to their specific experimental condition, each participant was asked to rate their feeling of well-being on a 5 -point Likert scale $(0=$ strongly disagree; $5=$ strongly agree). This was administered immediately before the scanning session and following the termination of the study while the subjects lay supine in the scanner.

Massage and its tactile control conditions

The three tactile stimulation conditions included a massage of the plantar surface of the foot with a wooden inanimate object, a Swedish foot massage or reflexology. The massage with the inanimate object served as a control for the human touch element of Swedish massage and reflexology conditions. The tool (obtained from the Kiné-Concept Institute of Ottawa) used for the massage with an object is modeled after a commonly used and commercially available massage therapy tool, the L-bar. This wooden tool fits comfortably in the hand and has a short head used for deeper pressure while the longer head is more suitable for gliding along larger muscles. The wooden massage therapy tool was used to roll over the plantar surface of the right foot using strokes designed to mimic that of the Swedish massage along with similar pressure, allowing one to distinguish the effects of human touch from the actual pattern of strokes.

The Swedish massage condition was performed utilizing maneuvers aimed at muscle relaxation by manipulating the soft tissues of the plantar surface of the right foot. Massage cream was applied to the foot in order to avoid friction and the massage was performed using moderate pressure (Diego et al. 2004) with gentle stroking and gliding maneuvers (i.e. effleurage, petrissage, longitudinal and transversal foulage, the latter two maneuvers consist of thumb sliding on tendons, ligaments or muscles with the goal of separating these tissues).

In the third tactile condition, a reflexology treatment was applied on the right plantar surface of the foot using basic techniques employed in this particular therapy (i.e. hook in and back up, finger and thumb walking). Reflexology practices can be traced as far back as the ancient Egyptian times (Kunz and Kunz 2007). In the $19^{\text {th }}$ century, scientists began studying the idea behind reflexes and their effect on the body whereby pressure applied on one part of the body would cause a reaction elsewhere (Kunz and Kunz 2007). However, the efficacy of reflexology as a form of foot massage has garnered mixed results and recent reviews point towards no conclusive evidence of its effectiveness for various medical conditions (Ernst et al. 2011; Kim et al. 2010). For the purpose of this study, given its qualitatively different sensory experience, the reflexology foot massage 
was simply used as a comparison condition relative to the better established Swedish foot massage.

The three tactile stimulation procedures were performed throughout the scanning session (i.e. during both rest and task blocks) by a registered massage therapist. Each tactile treatment was administered for a duration of $8.5 \mathrm{~min}$. Because of the pressure generated by these tactile treatments, participants' legs were strapped down to the scanner bed in order to minimize movement, and hence, reduce any motion artifacts in the brain images. In order to control for carry-over effects from the different tactile stimulation conditions, a betweengroups design was employed.

\section{Go/No Go task}

As part of the scanning procedure, a simple non-cognitively demanding task (Go/No Go) was used to provide some degree of normalization of participants' basal neural activity. Specifically, the block design procedure involved alternate periods of rest followed by task performance in order to help equalize mental basal states between subjects and over time. In particular, mind wandering is known to influence the activity of default mode network brain regions (the same areas assessed in this study). Another reason for including the cognitive task was to observe how subject's "de-activation" of neural activity was influenced by the massage. Specifically, the cingulate cortical brain regions that we presently assessed are normally quite active under resting states (i.e. default mode activity) but become less active (or de-activate) in response to a challenge or specific cognitive stimulus. Hence, an important question is whether massage influenced the resting state, as well as transition to a more heightened neural arousal state.

The Go/No Go block design procedure involved a presentation of letters, one at a time on a screen for a period of $125 \mathrm{~ms}$, with an inter-stimulus interval of $875 \mathrm{~ms}$. One half of the stimuli were ' $\mathrm{X}$ ' and the other half were other capital letters randomly selected from the remainder of the alphabet. The ' $\mathrm{X}$ ' and 'non- $\mathrm{X}$ ' stimuli were presented in random order. There were two types of Go/No Go conditions. In the 'respond to $X^{\prime}$ condition, the subject was instructed to press a button with the right index finger when an ' $\mathrm{X}$ ' was presented, and refrain from pressing for all other letters. In the 'respond to non-X' condition, the subject was instructed to refrain from pressing for $\mathrm{X}$ and to press for all other letters with the right index finger. Both Go/No Go conditions were presented in epochs of $27 \mathrm{~s}$ duration. Each Go/No Go epoch was preceded by a $3 \mathrm{~s}$ instruction epoch and followed by a $30 \mathrm{~s}$ rest epoch. During instruction epochs, the instruction 'Press for X' or 'Press for all letters except $\mathrm{X}$ ' was presented on the screen. During rest epochs, the word 'REST' was presented and the subject was not required to make any motor response. Within the scanning session there were four ' $\mathrm{X}$ ' and four 'non- $\mathrm{X}$ ' epochs, presented in a counterbalanced order, starting with the ' $X$ ' condition (see Fig. 1).

Stimuli generated on the E-Prime program were presented to the participant by a computer controlled projection system that delivered a visual stimulus to a rear-projection screen located at the entrance to the magnet bore. Stimuli were presented as white letters on a black background. A mirror on the head coil allowed subjects to observe the screen which projected these stimuli. The distance between the subject's eyes and the screen was approximately 6 feet. The scanning room and magnet bore were darkened to allow easy visualization of the experimental stimuli. Button-press responses were recorded via a MRI compatible fibre optic device (Lightwave Medical, Vancouver, British Columbia, Canada). Participants were instructed to press as quickly and correctly as possible and if they made a mistake, to continue without thinking about the mistakes.

Imaging

Imaging was performed using a $1.5 \mathrm{~T}$ Siemens Magnetom Symphony MR scanner ( 8 channel head coil) with the quantum gradient set (maximum amplitude $=30 \mathrm{mT} / \mathrm{m}$ and slew rate $=125 \mathrm{~T} / \mathrm{m} / \mathrm{s}$ ). The participants lay supine and their head was firmly secured using a custom head holder and positioned approximately parallel to the anterior commissure - posterior commissure (AC-PC) plane using external references. In order to control for movement artifacts from the massage procedure, the participant's legs were strapped securely to the imaging scanner bed. A conventional T1weighted spin echo localizer was acquired and used to prescribe a subsequent 3D FLASH (TR/TE $11.2 / 21 \mathrm{~ms}$, flip angle $60^{\circ}$, field of view (FOV) $26 \times 26 \mathrm{~cm}^{2}, 256 \times 256 \mathrm{ma}$ trix, slice thickness $5 \mathrm{~mm}$ ) volume acquisition used for further structural analyses.

The scanning session began with an initial rest epoch of $15 \mathrm{~s}$ to allow longitudinal magnetic relaxation ( $\mathrm{T} 1 \mathrm{effects}$ ) to stabilize. Images collected during this initial rest epoch were not included in the image analysis. Twenty-seven whole brain $\mathrm{T} 2 *$-weighted echo planar images per volume with BOLD contrast were obtained $(\mathrm{TR}=3000 \mathrm{~ms}, \mathrm{TE}=40 \mathrm{~ms}$, flip angle $90^{\circ}$, matrix: $64 \times 64$, FoV: $24 \times 24 \mathrm{~cm}$, slice thickness $5 \mathrm{~mm}$, bandwidth $62.5 \mathrm{kHz}$ ). A total of 167 functional images were collected in a total period of $501 \mathrm{~s}$.

Image processing

The fMRI data were processed and analyzed according to the general linear model procedure by Friston et al. (1995) as implemented in Statistical Parametric Mapping (SPM8), (http://www.fil.ion.ucl.ac.uk) running on MATLAB2009b (The Mathworks Inc., Natick, MA, USA).The functional images for each subject were corrected for motion artefacts 


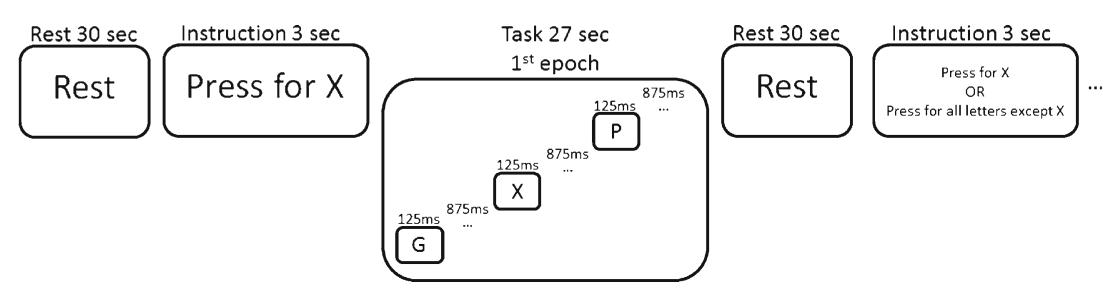

Fig. 1 The Go/No Go task lasted $8.5 \mathrm{~min}$. Participants were asked to rest for $30 \mathrm{~s}$ prior to the commencement of the task. All participants started with the "Press for X" epoch, whereby letters were presented for $125 \mathrm{~ms}$, followed by an inter stimulus interval of $875 \mathrm{~ms}$. This epoch lasted $27 \mathrm{~s}$

by realignment to the mean image. The realignment parameters were involved later in the design matrix as multiple regressors. Subject's T1-structural images were co-registered to the mean of the functional image.

The following step of spatial normalization was realized by warping the coregistered T1-structural image to the standard T1-weighted SPM template (Ashburner and Friston 1999) provided by the Montreal Neurological Institute (MNI). These parameters were then applied to all functional images. The normalized functional images were re-sampled to $2 \times 2 \times 2 \mathrm{~mm}$ and smoothed with a $10 \mathrm{~mm}$ full width at half-maximum Gaussian filter. The fMRI data were filtered using a high pass filter of $128 \mathrm{~s}$. A statistical model for each subject was computed by applying a canonical response function (Friston et al. 1998).

Imaging whole brain analysis

Voxel-based analyses were performed to assess global brain function changes without limiting ourselves to a priori regions of interest. The rest and Go/No Go task were included in the SPM model as independent factors. Regionally specific condition effects were tested by employing linear contrasts for each subject and each condition. The contrast images obtained from this design matrix (first level, fixed effects) were then taken to the second-level of analysis (random effects) using a one-sample t-test. To control for the multiple testing problem, we applied a family wise correction (Nichols and Hayasaka 2003).

The functional localizer approach (Saxe et al. 2006; Lamm and Decety 2008; Vul et al. 2009) was used to determine the brain region or a set of regions defined in each subject using a contrast targeting the regions specifically involved in the Rest condition as compared to the task (Rest $>$ Go/No Go). Following the recommendations made by Goldstein et al. (Goldstein et al. 2007), this contrast was calculated for the whole group of subjects $(n=40)$. This approach allowed for the exclusion of a significant influence of one of the groups on the determination of the regions of interest (ROI).

The anatomical localization of significant activations was assessed with reference to the standard stereotactic atlas by and was followed by a $30 \mathrm{~s}$ rest period. All task epochs (i.e. "Press for X" or "Press for all letters except X") were counterbalanced across participants and presented four times each, for a total of eight task blocks separated by $30 \mathrm{~s}$ rest periods

superimposition of the SPM maps on the standard SPM8 template. The main contrast (Rest $>\mathrm{Go} / \mathrm{No}$ Go) revealed significant signal changes in the subgenual anterior cingulate cortex (sACC: $x, y, z: 0,30,-2$ ) and the retrosplenial/ posterior cingulate cortex (RSC/PCC: $x, y, z: 14,-52,18$ ). Using the Marseille Region of Interest Toolbox software package (retrieved from: http://www.sourceforge.net/ projects/marsbar), signal changes in these ROIs were then extracted for each of the conditions, either during rest or during the Go/No Go task. For all statistical analyses performed, the positive affect subscale of the PANAS scale and reaction times for the 'non- $X$ ' condition across the first two trials were used as covariates (due to their significant differences between groups).

\section{Statistics}

Mean fMRI signal values from 0 to $21 \mathrm{~s}$ of the BOLD response for the rest and task contrasts were compared across groups using the statistical software program, PASW (IBM Corporation., Somers, NY, 2010). As such, a 2 (Time: Rest vs. Task) x 4 (Condition: control, object, massage, reflexology) mixed analysis of variance was performed to assess differences across the two different time points with respect to the different conditions. Tukey's HSD post-hoc tests were calculated where appropriate.

\section{Results}

\section{Behavioral data}

A 5-point Likert scale question (addressing current wellbeing) was posed by a member of the research team while the participant was positioned in the scanner awaiting study commencement, and once again immediately following the scanning session while still lying in the scanner. As such, each participant provided a pre- and post- measure of their current state of well-being (Fig. 2). Participants reported increased feelings of well-being immediately following the experiment $(F(1,36)=4.97, p=0.03$, Likert Pre: $\mathrm{M}=4.21$, 


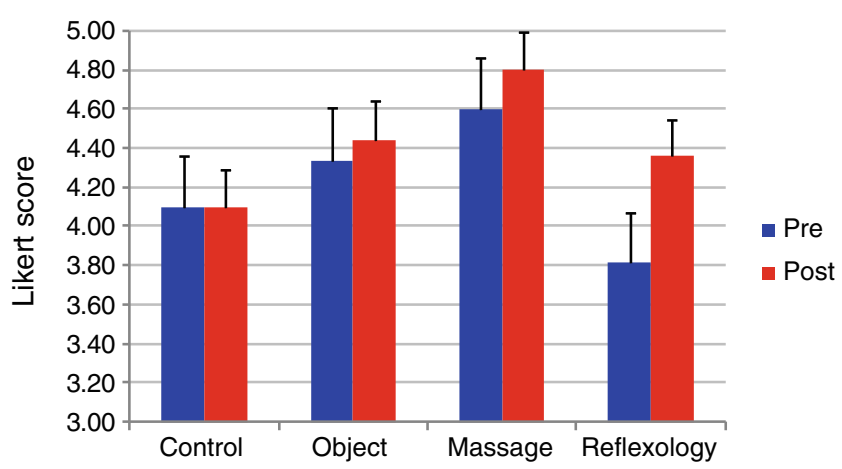

Fig. 2 Descriptive statistics showing the feeling of well-being felt before (Pre) and following (Post) the scanning session across groups. A score of 3 indicates neither agreement nor disagreement with the statement ("I am experiencing a pleasant feeling of well-being"), whereas a score of 5 indicates strong agreement

S.E.M. $=0.13$, Likert Post: $M=4.43$, S.E.M. $=0.10$ ), however, this effect did not differ across the treatment groups $(F(3,36)=2.23, p=0.10)$.

The BDI questionnaire served as a screening tool in order to ensure that participants did not exhibit depressive tendencies. As expected, there were no significant differences across treatment groups $\left(\chi^{2}(3)=5.20, p=0.16\right)$. In fact, the mean BDI score across groups was 2.73 (S.E.M. $=0.46$ ), indicating that, as expected, there was no indication of depression in this population.

The PANAS questionnaire was administered to participants prior to entering the fMRI scanner in order to determine if there were any pre-existing differences in positive and negative affect. Thus, we sought to ensure that participants that might score overly high or overly low on the PANAS would not preferentially accumulate in any particular treatment group. Surprisingly, significant differences were observed in the positive mood states $(F$ $(3,39)=3.13, p=0.04$; Fig. 3$)$, with participants that were

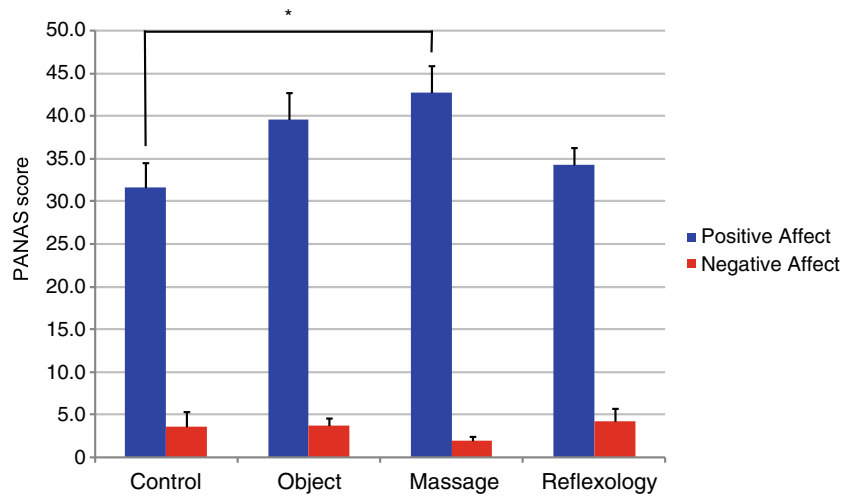

Fig. 3 Descriptive statistics showing the positive and negative subscale scores of the PANAS. Higher scores indicate a stronger association (i.e. strongly agree) whereas lower scores designate a discordance (i.e. strongly disagree) with the positive or negative affective adjectives on the PANAS to be assigned to the Swedish massage condition showing greater positive affect scores $(\mathrm{M}=4.27$, S.E.M. $=0.33)$ compared to the untreated control condition $(\mathrm{M}=3.17, \mathrm{~S}$. E.M. $=0.28$ ). As such, we used the positive affect scores as a covariate in our imaging analyses. No differences across groups were observed in the negative mood states $(F(3,39)=0.62, p=0.61)$.

Signal changes during the Go/No Go cognitive task and the resting state

The paradigm yielded the typical activation during a Go/ No Go cognitive task (see Supplementary Tables 1-3), with non-X trials showing greater activity in parietal and prefrontal cortical brain regions and $\mathrm{X}$ trials activating occipital and temporal regions. These data corroborate with previous findings outlining these brain regions as being responsible for decision making and monitoring processes in cognitive tasks involving response inhibition (Watanabe et al. 2002; Simmonds et al 2008; Liddle et al. 2001). Following this, we evaluated the resting state across our sample of 40 participants (Rest $>\mathrm{Go} / \mathrm{No}$ Go) in order to assess resting state activity in two prominent DMN regions, namely the posterior and anterior cingulate cortices. This contrast yielded significant brain clusters (Table 1), which were used as functional localizers. Signal changes were determined in these regions during the resting state across the four groups.

Signal changes in the subgenual anterior cingulate cortex (sACC)

During the rest blocks, participants displayed positive signal changes in the sACC $\left(x y z=0,30,-2, \mathrm{p}_{\mathrm{FWE}}<0.001\right)$ in the Control, Swedish massage and Reflexology groups, with the Swedish massage group showing the greatest overall positive BOLD response. The Go/No Go task evoked a deactivation across all groups (as indicated by a negative BOLD signal), which is typical of a default mode response to stimulus-induced activity (Fig. 4). No interaction was found between the Time (Rest vs. Go/No Go) x Treatment conditions $(\mathrm{F}(3,36)=0.92, p=0.44)$. However, a statistically significant main effect was observed for Time (i.e. Rest vs. $\mathrm{Go} / \mathrm{No} \mathrm{Go})(\mathrm{F}(1,36)=4.40, p=0.04$; Rest: $\mathrm{M}=0.02, \mathrm{SE}=0.04$; Task: $\mathrm{M}=-0.07, \mathrm{SE}=0.02$ ).

Signal changes in the retrosplenial/posterior cingulate cortex (RSC/PCC)

As was the case for the sACC, no significant Time $\mathrm{x}$ Treatment group interaction was apparent for the retrosplenial/ posterior cingulate cortex $(\mathrm{RSC} / \mathrm{PCC})(\mathrm{F}(3,36)=0.23, p=$ 0.88 ), however a statistically significant main effect of group 
Table 1 Activation clusters for Rest> Task (Go/No Go) contrast
BOLD responses are reported for clusters that surpassed an uncorrected threshold of $p<0.001$ and a corrected $p$-value of 0.05 on cluster level.

$\mathrm{MNI}=$ Montreal

Neurological Institute

*FWE correction, $p<0.05$

\begin{tabular}{|c|c|c|c|c|c|c|}
\hline \multirow[t]{2}{*}{ Anatomical location } & \multirow[t]{2}{*}{$p$ value } & \multirow[t]{2}{*}{$t$ value } & \multirow[t]{2}{*}{ Cluster size } & \multicolumn{3}{|c|}{$\begin{array}{l}\text { MNI coordinates } \\
\text { of max. } t \text { value }\end{array}$} \\
\hline & & & & $x$ & $y$ & $z$ \\
\hline Posterior cingulate (precuneus) & 0.000 & $16.34(\mathrm{Z}=\mathrm{inf})$ & 3715 & -4 & -58 & $24 *$ \\
\hline \multicolumn{7}{|l|}{ Retrosplenial/Posterior cingulate } \\
\hline (RSC/PCC) & 0.000 & $8.21(\mathrm{Z}=6.16)$ & & 14 & -52 & $18^{*}$ \\
\hline Angular gyrus & 0.000 & $7.26(Z=5.69)$ & 886 & -44 & -66 & $34^{*}$ \\
\hline Middle temporal gyrus & 0.000 & $7.26(Z=5.69)$ & & -46 & -72 & $22 *$ \\
\hline Middle temporal gyrus & 0.000 & $5.93(Z=4.94)$ & 404 & 48 & -62 & $22 *$ \\
\hline Middle temporal gyrus & 0.000 & $5.19(Z=4.47)$ & & 52 & -62 & 12 \\
\hline Lingual gyrus & 0.000 & $5.76(Z=4.84)$ & 186 & -2 & -88 & $-4^{*}$ \\
\hline Medial frontal gyrus & 0.000 & $5.45(Z=4.64)$ & 536 & -8 & 58 & 18 \\
\hline Medial frontal gyrus & 0.000 & $5.44(Z=4.63)$ & & -8 & 60 & 14 \\
\hline Medial frontal gyrus & 0.000 & $4.50(Z=3.99)$ & & -4 & 60 & \\
\hline Medial frontal gyrus & 0.000 & $4.46(Z=3.96)$ & & -12 & 52 & \\
\hline Medial frontal gyrus & 0.000 & $4.38(Z=3.91)$ & & -4 & 54 & -6 \\
\hline Fusiform gyrus & 0.000 & $5.35(Z=4.58)$ & 68 & -30 & -44 & -16 \\
\hline Superior temporal gyrus & 0.000 & $4.16(Z=3.74)$ & 101 & 60 & -20 & 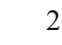 \\
\hline Superior temporal gyrus & 0.001 & $3.54(Z=3.26)$ & & 60 & -12 & -2 \\
\hline Superior temporal gyrus & 0.001 & $3.39(Z=3.15)$ & & 62 & -10 & \\
\hline Middle temporal gyrus & 0.001 & $3.38(Z=3.13)$ & & 60 & -10 & -8 \\
\hline Postcentral gyrus & 0.000 & $3.72(Z=3.41)$ & 6 & 58 & -20 & 30 \\
\hline Postcentral gyrus & 0.000 & $3.71(\mathrm{Z}=3.40)$ & 4 & 18 & -42 & 62 \\
\hline \multicolumn{7}{|l|}{ Subgenual anterior cingulate } \\
\hline cortex (sACC) & 0.000 & $3.68(\mathrm{Z}=3.37)$ & 24 & 0 & 30 & -2 \\
\hline Parahippocampal gyrus & 0.000 & $3.63(\mathrm{Z}=3.33)$ & 6 & 20 & -10 & -32 \\
\hline Insula (Heschl) & 0.001 & $3.41(Z=3.16)$ & 2 & 40 & -20 & 10 \\
\hline Inferior frontal gyrus & 0.001 & $3.40(Z=3.15)$ & 1 & -46 & 34 & -2 \\
\hline Fusiform gyrus & 0.001 & $3.38(Z=3.13)$ & 3 & 32 & -36 & -20 \\
\hline
\end{tabular}

in the $\mathrm{RSC} / \mathrm{PCC}(\mathrm{F}(3,39)=2.98, p=0.04$, global maxima: $\left.\mathrm{xyz}=44,-66,34, \mathrm{p}_{\mathrm{FWE}}<0.001\right)$, indicating a significant effect of time across all groups (Fig. 5). Importantly, in contrast to the sACC, there was no indication of task-induced deactivation during the Go/No Go cognitive task in the RSC/PCC in any of the treatment groups. contrast revealed an enhanced positive bold response signal

was observed $(\mathrm{F}(3,36)=3.72, p=0.02)$. As shown in Fig. 5 and confirmed by the post hoc comparisons, the Swedish massage group $(\mathrm{M}=0.12, \mathrm{SE}=0.02)$ had a significantly greater positive RSC/PCC BOLD response than the control group $(\mathrm{M}=0.02, \mathrm{SE}=0.02)$. Similarly, the Rest $>\mathrm{Go} /$ No Go task

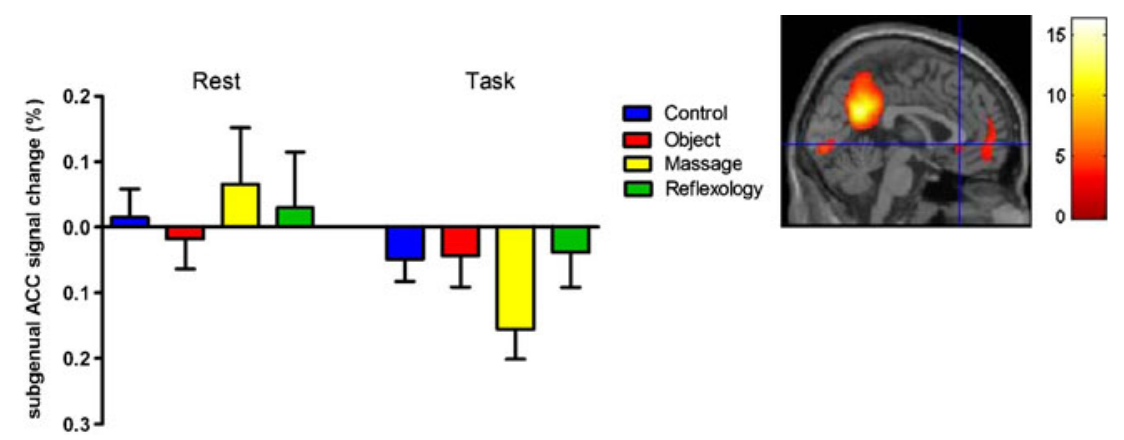

Fig. 4 Percent signal change in the subgenual anterior cingulate cortex (sACC, $x y z=0,30,-2$ ) during rest and in response to the Go/No Go cognitive association task across groups. SPM image shows the
Rest $>$ Go/No Go contrast, highlighting the sACC brain region $(p<0.05$, FWE-corrected). Error bars represent SEM 


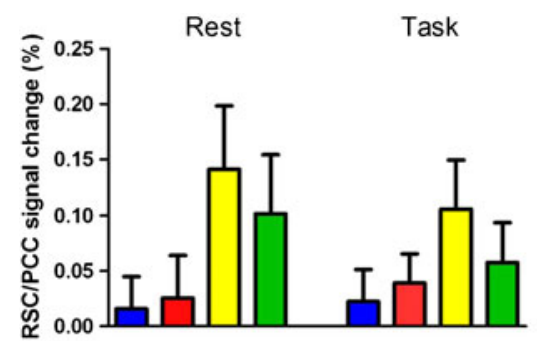

Fig. 5 Percent signal change in the retrosplenial/posterior cingulate cortex (RSC/PCC, $x y z=14,-52,18)$ during rest and in response to the Go/No Go cognitive task across groups. SPM image shows the Rest>

\section{Discussion}

Although massage has been widely applied for a variety of health reasons and substantial clinical reports indicate subjective benefits, virtually nothing is known regarding the neural systems affected by massage. Presently, we found that a single acute Swedish massage manipulation applied to the right plantar foot surface heightened resting state activation in two brain regions (sACC and RSC/PCC) that are important nodes in the default mode network (DMN); which is critical for self awareness and arousal. Performance of a cognitive task induced a deactivation of the BOLD signal in the SACC but not the RSC/ PCC. Furthermore, this de-activation response was not significantly modulated by the massage treatment. Hence, although acute Swedish massage increased resting state BOLD amplitude, it did not influence transitions from this state in response to an external stimulus. While the object massage did not appear to differ from control, the reflexology condition had modest consequences upon resting BOLD activity in the RSC/ PCC. Overall, qualitatively different types of acute massage differentially modulated activity in key regions of the DMN.

It was of particular interest that increased sACC activation was only apparent during the resting block, whereas the Swedish massage augmented RSC/PCC activity throughout the rest and task blocks. As will be discussed in the ensuing sections, this outcome would seem to suggest that the anterior and posterior portions of the cingulate cortex might have divergent roles in the context of massage, with the former more closely aligned with DMN functions and the latter underlying some alternate aspects of the treatment. In fact, besides being proposed as critical for generating the resting state and elements of consciousness, these brain regions have also been implicated in emotional processes and are known to be dysregulated with psychiatric pathology (Etkin et al. 2006; Wang et al. 2007). Moreover, these brain regions have also been shown to play a role in diverse processes that might be affected by massage, including the modulation of somatosensory information and episodic memory formation (Greicius et al. 2004; Hua et al. 2008).

The present study suggests that qualitatively different aspects of massage, such as the nature of human touch, can

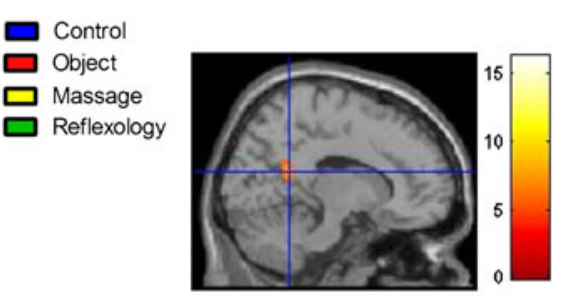

Go/No Go contrast, highlighting the RSC/PCC brain region $(p<0.05$, FWE-corrected). Error bars represent SEM

selectively modulate the activity of certain DMN brain regions. Indeed, the most robust fMRI changes were observed with the Swedish massage treatment, which involves long and smooth strokes with an applied pressure geared towards relaxation. The impact of reflexology, which is focused upon applying pressure to specific reflex points to invoke a beneficial response at distant body regions, was restricted to the $\mathrm{RSC} / \mathrm{PCC}$ brain region. In contrast, the massage with a wooden object, which involved pressure and strokes along the same areas of the foot as applied in the Swedish massage, had no significant effect on the BOLD signal in either of the brain regions. This latter finding is particularly noteworthy since it suggests the possibility that the human touch component (as opposed to the same pattern of massage with an object) had a profound influence upon the impact of the treatment. It is important to note that the massage with the object may not have activated the foot's muscle receptors as strongly as the reflexology and/or Swedish massage. Measurements with a pressure quantification system would have been helpful to determine if the intensity applied was similar to the other two massage conditions (Goldberg et al. 1992). Indeed, it has been observed that a moderate massage pressure, compared to light massage and vibratory stimulation, elicits a greater relaxation response (Diego et al. 2004).

Given that the greatest sACC activation was observed at rest with Swedish massage, it raises the possibility that the marked relaxation component that is expected to be unique to this treatment might underlie this effect (Lazar et al. 2000). Of interest, the ACC region has been shown to be involved in the affective representation, including the pleasantness of a stimulus (Rainville et al. 1997). Given that the reflexology massage was associated with the greatest change in the sense of well-being (at least as assessed using a Likert scale) after the treatment but did not affect the sACC, suggests that the differential changes in BOLD signal were not directly mirrored by emotional changes. However, the Likert scale is a relatively crude measure and would likely not detect subtle behavioral changes.

The present results are in agreement with the scant data that exist regarding the physiological effects of massage. In particular, using PET imaging, Ouchi et al. (2006) observed 
increased activation in the anterior cingulate cortex in response to an acute back massage treatment. Moreover, a recent fMRI study using vibrotactile stimulation on the plantar surface of the foot revealed increased activation of the inferior and posterior cingulate gyrus, along with the somatosensory cortex, insula, thalamus, caudate and cerebellum (Golaszewski et al. 2006). It was suggested that these brain regions integrate tactile sensory information from mechanoreceptors in the integumentary system with proprioceptive input from underlying muscles and joints. In effect, massage therapy would likely be affecting somatosensory regions directly, as well as modulating emotional regulatory cortical and limbic areas.

In addition to imaging studies, some animal research using tactile stimulation paradigms akin to those observed with massage, have begun to reveal a wide range of positive effects. Indeed, massage-like stroking in early life has been shown to influence brain (Guzzetta et al. 2009) and skeletal development in juvenile and young rats (Chen et al. 2009). Moreover, neonatal tactile stimulation has been shown to lead to long-term facilitative effects in cognition involving spatial working memory processes (Zhang and Cai 2008). Its effects are also noticeable with regards to reductions in gastrointestinal hormones like insulin and gastrin (Holst et al. 2005). However, increases in insulin secretion in response to sensory stimulation have also been noted (Uvnäs-Moberg 1987; Uvnäs-Moberg et al. 1987). The sensory stimulation provided by tactile stimulation has been shown to lower blood pressure and heart rate (Lund et al. 1999) which might contribute to the sedative effects observed with abdominal stroking in rats (Uvnäs-Moberg et al. 1996). In effect, one can speculate that massage therapy in humans might likewise provoke similar mechanisms.

\section{Limitations \& future directions}

Given the exploratory nature of this study, several caveats should be noted. Although our inclusion criteria required participants to have previous experience with massage from professional therapists, not everyone might have been exposed to foot reflexology or a massage with an object (which are far less common than Swedish massage). This may have elicited curiosity at the beginning of the scanning session. As well, another important limitation of this study includes the brevity of the massage treatments. While it is important to note that our aim was to assess the immediate effects of a single massage session on brain activity, this treatment is not representative of the relaxation one would experience in a conventional massage therapy setting. The scanning procedure was limited to under $20 \mathrm{~min}$ in order to reduce discomfort and any feelings of anxiety the participants may have felt while being in this restrictive environment. As such, the massage treatment was only applied to the right foot in order to obtain reliable indices of neural activity in response to the massage treatments. In this case, the duration of the experiment might not have been sufficient to detect long-term effects of the tactile stimulation conditions. Indeed, repeated massage sessions (30-60 $\mathrm{min}$ ) would have been optimal to capture any enduring positive neural and behavioral changes. Moreover, in order to maintain internal validity within our research design, a non-demanding cognitive Go/No Go association task was presented in block sequences during the massage treatments. As mentioned earlier, this was used to minimize extraneous mental processes between participants.

Future investigations should seek to determine how these brain regions are affected in conditions that have been associated with faulty default mode network regulation, including major depression (DeRubeis et al. 2008; Sheline et al. 2009). It should be considered that although such neural changes might be related to endogenous biological dispositional factors, it is also likely that the actual symptomatology in major depressive disorder, such as ruminations, apathy and sense of hopelessness ultimately give rise to disturbances in DMN connectivity (Veer et al. 2010). Ultimately, long term massage treatments may be a useful adjunct treatment to more traditional pharmacological and cognitive treatments aimed at modulating cingulate and prefrontal cortical regions that are important in the regulation and processing of emotional stimuli.

Acknowledgements The authors thank Glenn Walsh and Niall Duncan for assistance with data collection and processing.

Conflict of Interest Statement All authors declare that there are no conflicts of interest.

Open Access This article is distributed under the terms of the Creative Commons Attribution Noncommercial License which permits any noncommercial use, distribution, and reproduction in any medium, provided the original author(s) and source are credited.

\section{References}

Ashburner, J., \& Friston, K. J. (1999). Nonlinear spatial normalization using basis functions. Human Brain Mapping, 7(4), 254-266.

Barnes, P. M., Bloom, B., \& Nahin, R. L. (2008). Complementary and alternative medicine use among adults and children: United States, 2007. National health statistics reports; no 12. Hyattsville: National Center for Health Statistics.

Beck, A. T., Ward, C. H., Mendelson, M., Mock, J., \& Erbaugh, J. (1961). An inventory for measuring depression. Archives of General Psychiatry, 4, 53-63.

Beck, A. T., Steer, R. A., \& Garbin, M. G. (1988). Psychometric properties of the Beck Depression Inventory: twenty-five years of evaluation. Clinical Psychology Review, 8(1), 77-100. 
Beider, S., Mahrer, N. E., \& Gold, J. I. (2007). Pediatric massage therapy: an overview for clinicians. Pediatric Clinics of North America, 54(6), 1025-41.

Berlucchi, G., \& Aglioti, S. M. (2010). The body in the brain revisited. Experimental Brain Research, 200(1), 25-35.

Calenda, E. (2006). Massage therapy for cancer pain. Current Pain and Headache Reports, 10(4), 270-274.

Chen, H., Miller, S., Shaw, J., \& Moyer-Mileur, L. (2009). Massage therapy during early postnatal life promotes greater lean mass and bone growth, mineralization, and strength in juvenile and young adult rats. Journal of Musculoskeletal and Neuronal Interactions, 9(4), 278-287.

Craig, A. D. (2002). How do you feel? Interoception: the sense of the physiological condition of the body. Nature Reviews Neuroscience, 3(8), 655-66.

DeRubeis, R. J., Siegle, G. J., \& Hollon, S. D. (2008). Cognitive therapy versus medication for depression: treatment outcomes and neural mechanisms. Nature Reviews Neuroscience, 9(10), 788-796.

Diego, M. A., Field, T., Sanders, C., \& Hernandez-Reif, M. (2004). Massage therapy of moderate and light pressure and vibrator effects on EEG and heart rate. International Journal of Neuroscience, 114(1), 31-45.

Ernst, E., Posadzki, P., \& Lee, M. S. (2011). Reflexology: an update of a systematic review of randomised clinical trials. Maturitas, 68 (2), 116-20.

Etkin, A., Egner, T., Peraza, D. M., Kandel, E. R., \& Hirsch, J. (2006). Resolving emotional conflict: a role for the rostral anterior cingulate cortex in modulating activity in the amygdala. Neuron, 51(6), $871-82$

Field, T. (2002). Massage therapy. Medical Clinics of North America, $86(1), 163-171$

Field, T., Grizzle, N., Scafidi, F., \& Schanberg, S. (1996). Massage and relaxation therapies' effects on depressed adolescent mothers. Adolescence, 31(124), 903-911.

Field, T., Diego, M., Hernandez-Reif, M., Dieter, J., Kumar, A., Schanberg, S., et al. (2008). Insulin and Insulin-Like Growth Factor I (IGF-1) increase in preterm infants following massage therapy. Journal of Developmental and Behavioral Pediatrics, 29(6), 463-466.

Field, T., Diego, M., \& Hernandez-Reif, M. (2010). Preterm infant massage therapy research: a review. Infant Behavior \& Development, 33(2), 115-24.

Friston, K. J., Holmes, A. P., Worsley, K. J., Poline, J. B., Frith, C., \& Frackowiak, R. S. J. (1995). Statistical parametric maps in functional imaging: a general linear approach. Human Brain Mapping, 2(4), 189-210.

Friston, K. J., Fletcher, P., Josephs, O., Holmes, A., Rugg, M. D., \& Turner, R. (1998). Event-related fMRI: characterizing differential responses. NeuroImage, 7(1), 30-40.

Golaszewski, S. M., Siedentopf, C. M., Koppelstaetter, F., Fend, M., Ischebeck, A., Gonzalez-Felipe, V., et al. (2006). Human brain structures related to plantar vibrotactile stimulation: a functional magnetic resonance imaging study. NeuroImage, 29(3), 923-9.

Goldberg, J., Sullivan, S. J., \& Seaborne, D. E. (1992). The effect of two intensities of massage on H-reflex amplitude. Physical Therapy, 72(6), 449-57.

Goldstein, R. Z., Alia-Klein, N., Tomasi, D., Zhang, L., Cottone, L. A., Maloney, T., et al. (2007). Is decreased prefrontal cortical sensitivity to monetary reward associated with impaired motivation and self-control in cocaine addiction? The American Journal of Psychiatry, 164(1), 43-51.

Greicius, M. D., Srivastava, G., Reiss, A. L., \& Menon, V. (2004). Default-mode network activity distinguishes Alzheimer's disease from healthy aging: evidence from functional MRI. Proceedings of the National Academy of Sciences of the United States of America, 101(13), 4637-42.
Greicius, M. D., Kiviniemi, V., Tervonen, O., Vainionpää, V., Alahuhta, S., Reiss, A. L., et al. (2008). Persistent default-mode network connectivity during light sedation. Human Brain Mapping, 29(7), 839-847.

Guzzetta, A., Baldini, S., Bancale, A., Baroncelli, L., Ciucci, F., Ghirri, P., et al. (2009). Massage accelerates brain development and the maturation of visual function. Journal of Neuroscience, 29(18), 6042-6051.

Hart, S., Field, T., Hernandez-Reif, M., \& Lundy, B. (1998). Preschoolers' cognitive performance improves following massage. Early Child Development and Care, 143(1), 59-64.

Holst, S., Lund, I., Petersson, M., \& Uvnäs-Moberg, K. (2005). Massage-like stroking influences plasma levels of gastrointestinal hormones, including insulin, and increases weight gain in male rats. Autonomic Neuroscience, 120(1-2), 73-79.

Hou, W. H., Chiang, P. T., Hsu, T. Y., Chiu, S. Y., \& Yen, Y. C. (2010). Treatment effects of massage therapy in depressed people: a metaanalysis. The Journal of Clinical Psychiatry, 71(7), 894-901.

Hua, Q. P., Zeng, X. Z., Liu, J. Y., Wang, J. Y., Guo, J. Y., \& Luo, F. (2008). Dynamic changes in brain activations and functional connectivity during affectively different tactile stimuli. Cellular and Molecular Neurobiology, 28(1), 57-70.

Hughes, D., Ladas, E., Rooney, D., \& Kelly, K. (2008). Massage therapy as a supportive care intervention for children with cancer. Oncology Nursing Forum, 35(3), 431-42.

Johansen-Berg, H., Gutman, D. A., Behrens, T. E., Matthews, P. M., Rushworth, M. F., Katz, E., et al. (2008). Anatomical connectivity of the subgenual cingulate region targeted with deep brain stimulation for treatment-resistant depression. Cerebral Cortex, 18(6), 1374-83.

Jones, N. A., \& Field, T. (1999). Massage and music therapies attenuate frontal EEG asymmetry in depressed adolescents. Adolescence, 34 (135), 529-34.

Khalsa, S. S., Rudrauf, D., Feinstein, J. S., \& Tranel, D. (2009). The pathways of interoceptive awareness. Nature Neuroscience, 12 (12), 1494-496.

Kim, J. I., Lee, M. S., Kang, J. W., Choi do, Y., \& Ernst, E. (2010). Reflexology for the symptomatic treatment of breast cancer: a systematic review. Integrative Cancer Therapies, 9(4), 326-30.

Krohn, M., Listing, M., Tjahjono, G., Reisshauer, A., Peters, E., Klapp, B. F., et al. (2011). Depression, mood, stress, and Th1/Th2 immune balance in primary breast cancer patients undergoing classical massage therapy. Supportive Care in Cancer, 19(9), 1301-11.

Kunz, B., \& Kunz, K. (2007). Complete reflexology for life. New York: DK Publishing.

Lamm, C., \& Decety, J. (2008). Is the extrastriate body area (EBA) sensitive to the perception of pain in others? Cerebral Cortex, 18 (10), 2369-2373.

Lazar, S. W., Bush, G., Gollub, R. L., Fricchione, G. L., Khalsa, G., \& Benson, H. (2000). Functional brain mapping of the relaxation response and meditation. Neuroreport, 11(7), 1581-5.

Liddle, P. F., Kiehl, K. A., \& Smith, A. M. (2001). Event-related fMRI study of response inhibition. Human Brain Mapping, 12(2), 100-9.

Lund, I., Lundeberg, T., Kurosawa, M., \& Uvnäs-Moberg, K. (1999). Sensory stimulation (massage) reduces blood pressure in unanaesthetized rats. Journal of the Autonomic Nervous System, 78(1), 30-37.

Moyer, C. A., Rounds, J., \& Hannum, J. W. (2004). A meta-analysis of massage therapy research. Psychological Bulletin, 130(1), 3-18.

Nahin, R. L., Barnes, P. M., Stussman, B. J., \& Bloom, B. (2009). Costs of complementary and alternative medicine (CAM) and frequency of visits to CAM practitioners: United States, 2007. National health statistics reports; no 18. Hyattsville: National Center for Health Statistics.

Nichols, T., \& Hayasaka, S. (2003). Controlling the familywise error rate in functional neuroimaging: a comparative review. Statistical Methods in Medical Research, 12(5), 419-446. 
Northoff, G., Qin, P., \& Nakao, T. (2010). Rest-stimulus interaction in the brain: a review. Trends in Neurosciences, 33(6), 277-84.

Ouchi, Y., Kanno, T., Okada, H., Yoshikawa, E., Shinke, T., Nagasawa, S., et al. (2006). Changes in cerebral blood flow under the prone condition with and without massage. Neuroscience Letters, 407(2), 131-5.

Park, J. (2005). Use of Alternative Health Care. Health Reports, 16(2), $39-42$.

Procianoy, R. S., Mendes, E. W., \& Silveira, R. C. (2010). Massage therapy improves neurodevelopment outcome at two years corrected age for very low birth weight infants. Early Human Development, 86(1), 7-11.

Raichle, M. E., MacLeod, A. M., Snyder, A. Z., Powers, W. J., Gusnard, D. A., \& Shulman, G. L. (2001). A default mode of brain function. Proceedings of the National Academy of Sciences of the United States of America, 98, 676-682.

Rainville, P., Duncan, G. H., Price, D. D., Carrier, B., \& Bushnell, M. C. (1997). Pain affect encoded in human anterior cingulate but not somatosensory cortex. Science, 277(5328), 968-71.

Rapaport, M. H., Schettler, P., \& Bresee, C. (2010). A preliminary study of the effects of a single session of Swedish massage on hypothalamicpituitary-adrenal and immune function in normal individuals. Journal of Alternative and Complementary Medicine, 10, 1-10.

Russell, N. C., Sumler, S. S., Beinhorn, C. M., \& Frenkel, M. A. (2008). Role of massage therapy in cancer care. Journal of Alternative and Complementary Medicine, 14(2), 209-14.

Saxe, R., Brett, M., \& Kanwisher, N. (2006). Divide and conquer: a defense of functional localizers. NeuroImage, 30(4), 1088-1099.

Schneider, F., Bermpohl, F., Heinzel, A., Rotte, M., Walter, M., Tempelmann, C., et al. (2008). The resting brain and our self: selfrelatedness modulates resting state neural activity in cortical midline structures. Neuroscience, 157(1), 120-31.

Sheline, Y. I., Barch, D. M., Price, J. L., Rundle, M. M., Vaishnavi, S. N., Snyder, A. Z., et al. (2009). The default mode network and selfreferential processes in depression. Proceedings of the National Academy of Sciences of the United States of America, 106(6), 1942-7.

Sherman, K. J., Cherkin, D. C., Hawkes, R. J., Miglioretti, D. L., \& Deyo, R. A. (2009). Randomized trial of therapeutic massage for chronic neck pain. The Clinical Journal of Pain, 25(3), 233-238.

Simmonds, D. J., Pekar, J. J., \& Mostofsky, S. H. (2008). Metaanalysis of Go/No-go tasks demonstrating that fMRI activation associated with response inhibition is task-dependent. Neuropsychologia, 46(1), 224-232.

Taylor, K. S., Seminowicz, D. A., \& Davis, K. D. (2009). Two systems of resting state connectivity between the insula and cingulate cortex. Human Brain Mapping, 30(9), 2731-45.
Uvnäs-Moberg, K. (1987). Gastrointestinal hormones and pathophysiology of functional gastrointestinal disorders. Scandinavian Journal of Gastroenterology, 128, 138-46.

Uvnäs-Moberg, K., Widstrom, A. M., Marchini, G., \& Winberg, J. (1987). Release of GI Hormones in Mother and Infant by Sensory Stimulation. Acta Paediatrica Scandinavica, 76(6), $851-860$.

Uvnäs-Moberg, K., Alster, P., Lund, I., Lundeberg, T., Kurosawa, M., \& Ahlenius, S. (1996). Stroking of the abdomen causes decreased locomotor activity in conscious male rats. Physiology and Behavior, 60(6), 1409-1411.

Vanhaudenhuyse, A., Noirhomme, Q., Tshibanda, L. J., Bruno, M. A., Boveroux, P., Schnakers, C., et al. (2010). Default network connectivity reflects the level of consciousness in non-communicative brain-damaged patients. Brain, 133(Pt 1), 161-171.

Vanhaudenhuyse, A., Demertzi, A., Schabus, M., Noirhomme, Q., Bredart, S., Boly, M., et al. (2011). Two distinct neuronal networks mediate the awareness of environment and of self. Journal of Cognitive Neuroscience, 23(3), 570-578.

Veer, I. M., Beckmann, C. F., van Tol, M. J., Ferrarini, L., Milles, J., Veltman, D. J., et al. (2010). Whole brain resting-state analysis reveals decreased functional connectivity in major depression. Frontiers in Systems Neuroscience, 4, 41-51.

Vul, E., Harris, C., Winkielman, P., \& Pashler, H. (2009). Puzzling high correlations in fMRI studies of emotion, personality and social cognition. Perspectives on Psychological Science, 4(3), 274-290.

Wang, L., Hosakere, M., Trein, J. C., Miller, A., Ratnanather, J. T., Barch, D. M., et al. (2007). Abnormalities of cingulate gyrus neuroanatomy in schizophrenia. Schizophrenia Research, 93(13), 66-78.

Watanabe, J., Sugiura, M., Sato, K., Sato, Y., Maeda, Y., Matsue, Y., et al. (2002). The human prefrontal and parietal association cortices are involved in NO-GO performances: an event-related fMRI study. NeuroImage, 17(3), 1207-1216.

Watson, D., Clark, L. A., \& Tellegen, A. (1988). Development and validation of brief measures of positive and negative affect: The PANAS scales. Journal of Personality and Social Psychology, 54 (6), 1063-1070.

Wiens, S. (2005). Interoception in emotional experience. Current Opinion in Neurology, 18(4), 442-7.

Zhang, M., \& Cai, J. X. (2008). Neonatal tactile stimulation enhances spatial working memory, prefrontal long-term potentiation, and D1 receptor activation in adult rats. Neurobiology of Learning and Memory, 89(4), 397-406. 